\title{
Simultaneous Target Detection and Multi-user Communications Enabled by Joint Beamforming
}

\author{
Fan Liu*†, Christos Masouros ${ }^{\dagger}$, Ang $\mathrm{Li}^{\dagger}$, Jianming Zhou* and Lajos Hanzo \\ * School of Information and Electronics, Beijing Institute of Technology, Beijing, China \\ ${ }^{\dagger}$ Department of Electronic and Electrical Engineering, University College London, London, UK \\ ${ }^{\ddagger}$ School of Electronics and Computer Science, University of Southampton, Southampton, UK \\ Email: liufan92@bit.edu.cn, chris.masouros@ieee.org, ang.li.14@ucl.ac.uk, zhoujm@bit.edu.cn, lh@ecs.soton.ac.uk
}

\begin{abstract}
In this paper, we propose several joint beamforming designs for the multi-input-multi-output (MIMO) dual-functional radar-communication system. We firstly split the antennas into two groups, one for radar and the other for communication. With this separated deployment, the radar signal is designed to fall into the null-space of the downlink communication channel. The communication beamformer is optimized such that the obtained beampattern matches the radar's beampattern while satisfying the performance requirements of the downlink users. We further consider a second operational option, where all the antennas transmit a joint waveform that is shared by both radar and communications, and formulate an appropriate beampattern while guaranteeing the downlink cellular transmission. Numerical results show that both methods are able to realize the simultaneous radar detection and wireless information transfer.
\end{abstract}

\section{INTRODUCTION}

As one of the most valuable resources, the radio frequency spectrum is now in great demand because of the exponentially growing wireless services and devices. Currently, policy regulators and network providers are seeking the possibility for sharing the radar bands with communication users [1], [2], which has been widely recognized as an enabler for the efficient usage of the spectrum in the future. In [3], an opportunistic spectrum sharing approach has been proposed, where the communication system transmits signals when the frequency and spatial spectra are not occupied by the radar. While such a technique is straightforward and easy to implement, it does not allow the radar and communication systems to operate simultaneously. In view of this, a nullspace projection (NSP) method has been studied by [4], in which a linear precoder is designed to project the radar signal onto the null-space of the interference channel matrix between the radar and the base station (BS), thus zero-forcing the interfering signal generated by the radar. Further, optimizationbased techniques have been considered in [5], [6] to provide a controllable trade-off between point-to-point (P2P) MIMO communication system and the MIMO radar, which maximize the performance metrics of one system under the constraints of the other. To address the coexistence scenario of radar and multi-user MIMO (MU-MIMO) communications, a radarspecific beamforming design has been proposed by [7], where the detection probability of radar is maximized under the SINR and power constraints of the communications. This technique has been further expanded in [8] by exploiting the multi-user interference (MUI) as a green signal power source, which offers significant power-savings compared to the conventional schemes.

While the above spectrum sharing methods are welldesigned, one critical drawback is that they typically require to exchange side information between the two systems, e.g., radar probing waveform, communication modulation formats and channel state information (CSI), which is hard to be implemented in realistic scenarios. Therefore, a more favorable solution is to carry out both radar and communication operations by designing a dual-functional system, where the above shortfalls can be avoided. Existing research efforts focus on the waveform design for MIMO radar-communication (RadCom) systems, where the information bits are modulated by varying the sidelobe levels of the probing beampattern [9], or by shuffling the waveforms across the antennas [10]. It is worth highlighting that in such schemes, a communication symbol is represented by one or several radar pulses, and hence leads to a low data rate, which is at the same order-of-magnitude with the pulse repetition frequency (PRF) of the radar [10]. More recently, a waveform design approach has been proposed by [11], which relies on a spatial division multiple-access (SDMA) framework to formulate the transmit beams in two different directions for communication and radar, respectively. Unfortunately, it is obvious that this technique can only be used for Line-of-Sight (LoS) communications, and fails to address the more complicated case of communications in the fading channels.

In this paper, we consider the beamforming designs for the MIMO RadCom system, which allow simultaneous target detection and downlink communications with multiple users in fading channels. Unlike the conventional approaches mentioned above [9], [10], we design the beamforming matrices rather than the concrete waveforms, and thus avoiding the changes in the modulation scheme as well as the low transmission rate of the communication system. Inspired by the NSP methods, a separated antenna deployment is proposed firstly, where we partition the transmit antennas into radar antennas and communication antennas. The radar interference imposed on the downlink users is canceled by enforcing zeroforcing constraints, and the communication covariance matrix is designed to formulate a beampattern that matches the radar beampattern, while guaranteeing the downlink SINR and the 


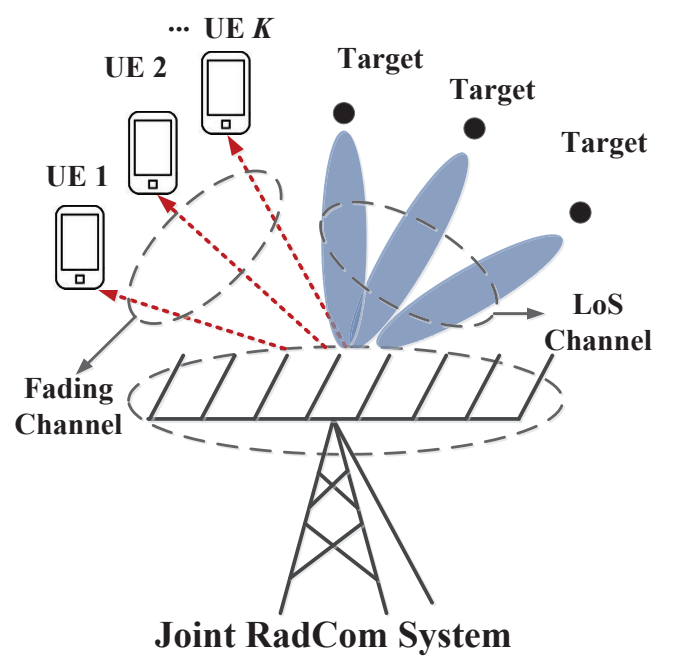

Fig. 1. Joint MIMO RadCom System

transmit power budget. As a step further, we propose a shared antenna deployment, where all the transmit antennas are jointly exploited by both radar and communication operations to formulate a probing beampattern while guaranteeing the downlink communication performance, which takes full advantage of the degrees of freedom (DoFs) of the system. The proposed optimization problems are non-convex, but can be reformulated as Semidefinite Programming (SDP) and solved by Semidefinite Relaxation (SDR) techniques. Numerical results verify the effectiveness of the proposed methods for both radar and communication performance.

\section{System ModeL}

We consider a joint MIMO RadCom system, which can simultaneously transmit probing signals to the targets located at the angles of interest and communication symbols to downlink users. As shown in Fig.1, the joint system is equipped with a uniform linear array (ULA) of $N$ antennas, serving $K$ single-antenna downlink users while detecting targets at the same time. In contrast to the scenario in [11], we assume that multiple users are in the fading channel rather than the LoS channel, which is more typical in realistic scenarios. Below we present the signal models for both of operations considered in this paper, namely the separated deployment and the shared deployment.

\section{A. Separated Deployment}

The separated deployment involves splitting the antenna array into two groups: one for radar and one for the downlink communications. In this case, the received signal of the $i$-th user is given by

$$
x_{i}[l]=\mathbf{g}_{i}^{T} \sum_{k=1}^{K} \mathbf{w}_{k} d_{k}[l]+\mathbf{f}_{i}^{T} \mathbf{s}_{l}+\omega_{i}[l], \forall i,
$$

where $\mathbf{g}_{i} \in \mathbb{C}^{N_{C} \times 1}, \mathbf{f}_{i} \in \mathbb{C}^{N_{R} \times 1}$ are the channel vectors from communication antennas and radar antennas to the $i$ th user, $N_{C}$ and $N_{R}$ are the number of antennas dedicated to communication and radar respectively, $d_{i}[l]$ and $\omega_{i}[l]$ stand for the communication symbol and the received noise of the $i$-th user at the time index $l$ both of which follow a complex Gaussian distribution with zero mean and variance $N_{0}, \mathbf{w}_{i} \in \mathbb{C}^{N_{C} \times 1}$ denotes the beamforming vector of the $i$ th user, and finally $\mathbf{s}_{l} \in \mathbb{C}^{N_{R} \times 1}$ is the $l$-th snapshot across the radar antennas. The sample covariance matrix of the radar signal is given as $\frac{1}{L} \sum_{l=1}^{L} \mathbf{s}_{l} \mathbf{s}_{l}^{H}=\mathbf{R}_{1} \in \mathbb{C}^{N_{R} \times N_{R}}$, with $L$ being the length of the signal on the fast-time axis. We rely on the following standard assumptions:

1) The communication signals are statistically independent of the radar signals;

2) The channel between the RadCom system and users is flat Rayleigh fading, which is given by $\mathbf{H}=$ $\left[\mathbf{h}_{1}, \mathbf{h}_{2}, \ldots, \mathbf{h}_{K}\right]$, where $\mathbf{h}_{i}=\left[\mathbf{f}_{i} ; \mathbf{g}_{i}\right] \in \mathbb{C}^{N \times 1}$. It is assumed that the channel is perfectly estimated by pilot symbols.

Note that the separated deployment allows arbitrary radar signals to be used.

With the denotation $\mathbf{W}_{k}=\mathbf{w}_{k} \mathbf{w}_{k}^{H}$, the communication transmit power is given as

$$
P_{1}=\sum_{k=1}^{K}\left\|\mathbf{w}_{k}\right\|^{2}=\sum_{k=1}^{K} \operatorname{tr}\left(\mathbf{W}_{k}\right) .
$$

Based on (1), the received SINR of the $i$-th user is thus given by

$$
\begin{aligned}
\beta_{i} & =\frac{\left|\mathbf{g}_{i}^{T} \mathbf{w}_{i}\right|^{2}}{\sum_{\substack{k=1 \\
k \neq i}}^{K}\left|\mathbf{g}_{i}^{T} \mathbf{w}_{k}\right|^{2}+\mathbf{f}_{i}^{T} \mathbf{R}_{1} \mathbf{f}_{i}^{*}+N_{0}} \\
= & \frac{\operatorname{tr}\left(\mathbf{g}_{i}^{*} \mathbf{g}_{i}^{T} \mathbf{W}_{i}\right)}{\operatorname{tr}\left(\mathbf{g}_{i}^{*} \mathbf{g}_{i}^{T} \sum_{\substack{k=1 \\
k \neq i}}^{K} \mathbf{W}_{k}\right)+\operatorname{tr}\left(\mathbf{f}_{i}^{*} \mathbf{f}_{i}^{T} \mathbf{R}_{1}\right)+N_{0}} .
\end{aligned}
$$

The covariance matrix for the precoded communication symbols can be obtained by

$$
\mathbf{C}_{1}=\sum_{k=1}^{K} \mathbf{W}_{k} .
$$

\section{B. Shared Deployment}

In this case, all the $N$ antennas are shared for both radar detection and downlink cellular transmission. The received signal at the $i$-th user is given as

$$
y_{i}[l]=\mathbf{h}_{i}^{T} \sum_{k=1}^{K} \mathbf{t}_{k} d_{k}[l]+n_{i}[l], \forall i,
$$

where $\mathbf{t}_{i} \in \mathbb{C}^{N \times 1}$ and $n_{i}[l] \sim \mathcal{C N}\left(0, N_{0}\right)$ denote the beamforming vector and the received noise of the $i$-th user, respectively. We make the following assumptions:

1) The RadCom system employs the communication signal as the radar probing waveform; 
2) As above, the channel $\mathbf{H}$ between the RadCom system and the users is Rayleigh flat fading and it is also perfectly estimated.

At first glance, it seems that there are no DoFs in designing the radar signal, since the communication signal is employed as a dual-functional waveform in the shared deployment. Nevertheless, we will show in the following sections that this is indeed an affordable constraint, given the resultant benefits. Similarly, the transmit power is given by

$$
P_{0}=\sum_{k=1}^{K}\left\|\mathbf{t}_{k}\right\|^{2}=\sum_{k=1}^{K} \operatorname{tr}\left(\mathbf{T}_{k}\right),
$$

where $\mathbf{T}_{k}=\mathbf{t}_{k} \mathbf{t}_{k}^{H}$. The received SINR of the $i$-th user is given by

$$
\gamma_{i}=\frac{\left|\mathbf{h}_{i}^{T} \mathbf{t}_{i}\right|^{2}}{\sum_{\substack{k=1 \\ k \neq i}}^{K}\left|\mathbf{h}_{i}^{T} \mathbf{t}_{k}\right|^{2}+N_{0}}=\frac{\operatorname{tr}\left(\mathbf{h}_{i}^{*} \mathbf{h}_{i}^{T} \mathbf{T}_{i}\right)}{\operatorname{tr}\left(\mathbf{h}_{i}^{*} \mathbf{h}_{i}^{T} \sum_{\substack{k=1 \\ k \neq i}}^{K} \mathbf{T}_{k}\right)+N_{0}} .
$$

Note that in (7) no radar interference is imposed on the users. The covariance matrix of the precoded symbols for the shared deployment is given by

$$
\mathbf{C}_{2}=\sum_{k=1}^{K} \mathbf{T}_{k}
$$

\section{Proposed Beamforming Designs}

In the proposed scenario, an appropriate beamformer should be designed to meet the following requirements:

- To generate a beampattern that is closely matched to the desired beampattern for radar detection;

- To guarantee the SINR level required for the downlink users;

- To satisfy the transmit power budget.

In this section, we first recall the beampattern designs for the colocated MIMO radar only, and then combine them with communication constraints to ensure that the beamformer obtained can indeed meet the above criteria.

\section{A. MIMO Radar Beampattern Design}

It is widely exploited that by employing uncorrelated waveforms, MIMO radar achieves higher DoFs than the traditional phased-array radar [12], [13]. The existing literature indicates that the design of such a beampattern is equivalent to designing the covariance matrix of the probing signals, where convex optimization can be employed. In [14], a constrained leastsquares problem is formulated to approach an ideal beampattern. Here we recapture it as the following optimization

$$
\begin{array}{ll}
\min _{\alpha, \mathbf{R}} & \sum_{m=1}^{M}\left|\alpha \tilde{P}_{d}\left(\theta_{m}\right)-\mathbf{a}^{H}\left(\theta_{m}\right) \mathbf{R a}\left(\theta_{m}\right)\right|^{2} \\
\text { s.t. } & \operatorname{diag}(\mathbf{R})=\frac{P_{0} \mathbf{1}}{N_{t}}, \\
& \quad \mathbf{R} \succeq 0, \mathbf{R}=\mathbf{R}^{H}, \\
& \alpha \geq 0,
\end{array}
$$

where $\left\{\theta_{m}\right\}_{m=1}^{M}$ is defined as a fine angular grid that covers the detection angle range of $[-\pi / 2, \pi / 2], \mathbf{a}\left(\theta_{m}\right)=$ $\left[1, e^{j 2 \pi \Delta \sin \left(\theta_{m}\right)}, \ldots, e^{j 2 \pi\left(N_{t}-1\right) \Delta \sin \left(\theta_{m}\right)}\right]^{T} \in \mathbb{C}^{N \times 1}$ is the steering vector of the transmit antenna array with $\Delta$ being the spacing between adjacent elements normalized by the wavelength, $N_{t}$ is the number of antennas of the array, $\tilde{P}_{d}\left(\theta_{m}\right)$ is the desired ideal beampattern gain at $\theta_{m}, \mathbf{R}$ is the waveform covariance matrix, $P_{0}$ is the power budget, $\alpha$ is a scaling factor, and $\mathbf{1}$ is defined as $\mathbf{1}=[1,1, \ldots, 1]^{T} \in \mathbb{R}^{N \times 1}$. The constraint (9b) is imposed to guarantee that the waveform transmitted by different antennas has the same average power.

Aimed to generate a beampattern with a desired $3 \mathrm{~dB}$ mainbeam width, another optimization problem has been proposed by Stoica et al. [12], which is given by

$$
\begin{array}{ll}
\min _{t, \mathbf{R}} & -t \\
s . t . & \mathbf{a}^{H}\left(\theta_{0}\right) \mathbf{R a}\left(\theta_{0}\right)-\mathbf{a}^{H}\left(\theta_{m}\right) \mathbf{R a}\left(\theta_{m}\right) \geq t, \forall \theta_{m} \in \Omega, \\
& \mathbf{a}^{H}\left(\theta_{1}\right) \mathbf{R a}\left(\theta_{1}\right)=\mathbf{a}^{H}\left(\theta_{0}\right) \mathbf{R a}\left(\theta_{0}\right) / 2, \\
& \mathbf{a}^{H}\left(\theta_{2}\right) \mathbf{R a}\left(\theta_{2}\right)=\mathbf{a}^{H}\left(\theta_{0}\right) \mathbf{R a}\left(\theta_{0}\right) / 2, \\
& \mathbf{R} \succeq 0, \mathbf{R}=\mathbf{R}^{H}, \\
& \operatorname{diag}(\mathbf{R})=\frac{P_{0} \mathbf{1}}{N_{t}},
\end{array}
$$

where $\theta_{0}$ is the location of the main-beam, $\left(\theta_{2}-\theta_{1}\right)$ determines the $3 \mathrm{~dB}$ main-beam width, and $\Omega$ denotes the sidelobe region. Note that the above two problems are convex, and thus can be efficiently solved by numerical tools.

\section{B. Zero-forcing Beamforming for Separated Deployment}

We first consider the beamforming design of the separated deployment. Motivated by the NSP method that has been widely applied to radar and communication co-existence scenarios [4], [15], [16], we force the radar signals to fall into the null-space of the channel between the radar antennas and downlink users to eliminate the interference. This can be equivalently written as

$$
\mathbb{E}\left\{\left\|\mathbf{f}_{i}^{T} \mathbf{s}_{l}\right\|^{2}\right\}=\mathbf{f}_{i}^{T} \mathbb{E}\left\{\mathbf{s}_{l} \mathbf{s}_{l}^{H}\right\} \mathbf{f}_{i}^{*}=\operatorname{tr}\left(\mathbf{f}_{i}^{*} \mathbf{f}_{i}^{T} \mathbf{R}_{1}\right)=0, \forall i .
$$

By introducing the above constraint in (9) and (10), and using $N_{R}$ antennas for radar detection, an $N_{R} \times N_{R}$ covariance matrix $\mathbf{R}_{1}$ can be obtained. Accordingly, the zero-forcing version for (9) and (10) are given by

$$
\begin{array}{ll}
\min _{\alpha, \mathbf{R}_{1}} & \sum_{m=1}^{M}\left|\alpha \tilde{P}_{d}\left(\theta_{m}\right)-\mathbf{a}^{H}\left(\theta_{m}\right) \mathbf{R a}\left(\theta_{m}\right)\right|^{2} \\
\text { s.t. } & \operatorname{diag}\left(\mathbf{R}_{1}\right)=\frac{P_{R} \mathbf{1}}{N} \\
& \mathbf{R}_{1} \succeq 0, \mathbf{R}_{1}=\mathbf{R}_{1}^{H}, \\
& \alpha \geq 0 \\
& \operatorname{tr}\left(\mathbf{f}_{i}^{*} \mathbf{f}_{i}^{T} \mathbf{R}_{1}\right)=0, \forall i,
\end{array}
$$




$$
\begin{aligned}
\min _{t, \mathbf{R}_{1}}-t & \\
\text { s.t. } & \mathbf{a}_{1}^{H}\left(\theta_{0}\right) \mathbf{R}_{1} \mathbf{a}_{1}\left(\theta_{0}\right)-\mathbf{a}_{1}^{H}\left(\theta_{m}\right) \mathbf{R}_{1} \mathbf{a}_{1}\left(\theta_{m}\right) \geq t, \forall \theta_{m} \in \Omega, \\
& \mathbf{a}_{1}^{H}\left(\theta_{1}\right) \mathbf{R}_{1} \mathbf{a}_{1}\left(\theta_{1}\right)=\mathbf{a}_{1}^{H}\left(\theta_{0}\right) \mathbf{R}_{1} \mathbf{a}_{1}\left(\theta_{0}\right) / 2, \\
& \mathbf{a}_{1}^{H}\left(\theta_{2}\right) \mathbf{R}_{1} \mathbf{a}_{1}\left(\theta_{2}\right)=\mathbf{a}_{1}^{H}\left(\theta_{0}\right) \mathbf{R}_{1} \mathbf{a}_{1}\left(\theta_{0}\right) / 2, \\
& \mathbf{R}_{1} \succeq 0, \mathbf{R}_{1}=\mathbf{R}_{1}^{H}, \\
& \operatorname{diag}\left(\mathbf{R}_{1}\right)=\frac{P_{R} \mathbf{1}}{N}, \\
& \operatorname{tr}\left(\mathbf{f}_{i}^{*} \mathbf{f}_{i}^{T} \mathbf{R}_{1}\right)=0, \forall i,
\end{aligned}
$$

respectively, where $\mathbf{a}\left(\theta_{m}\right)=\left[\mathbf{a}_{1}\left(\theta_{m}\right) ; \mathbf{a}_{2}\left(\theta_{m}\right)\right], \forall m$, and $\mathbf{a}_{1}\left(\theta_{m}\right) \in \mathbb{C}^{N_{R} \times 1}, \mathbf{a}_{2}\left(\theta_{m}\right) \in \mathbb{C}^{N_{C} \times 1}, \forall m, P_{R}$ is the transmission power for radar.

Since we assume that the transmitted signals for radar and communication are statistically independent, by recalling (4), it can be easily proven that the overall covariance matrix can be written as

$$
\tilde{\mathbf{C}}=\left[\begin{array}{cc}
\mathbf{R}_{1} & \mathbf{0} \\
\mathbf{0} & \mathbf{C}_{1}
\end{array}\right]=\left[\begin{array}{cc}
\mathbf{R}_{1} & \mathbf{0} \\
\mathbf{0} & \sum_{k=1}^{K} \mathbf{W}_{k}
\end{array}\right] .
$$

The beampattern gain at $\theta_{m}$ can be obtained as

$$
\begin{aligned}
P_{d}\left(\theta_{m}\right) & =\mathbf{a}^{H}\left(\theta_{m}\right) \tilde{\mathbf{C}} \mathbf{a}\left(\theta_{m}\right) \\
& =\mathbf{a}_{1}^{H}\left(\theta_{m}\right) \mathbf{R}_{1} \mathbf{a}_{1}\left(\theta_{m}\right)+\mathbf{a}_{2}^{H}\left(\theta_{m}\right) \sum_{k=1}^{K} \mathbf{W}_{k} \mathbf{a}_{2}\left(\theta_{m}\right) .
\end{aligned}
$$

If the shape of the overall beampattern perfectly matches the radar-only beampattern obtained by (12) and (13), we have

$$
\mathbf{a}_{2}^{H}\left(\theta_{m}\right) \sum_{k=1}^{K} \mathbf{W}_{k} \mathbf{a}_{2}\left(\theta_{m}\right)=\sigma \mathbf{a}_{1}^{H}\left(\theta_{m}\right) \mathbf{R}_{1} \mathbf{a}_{1}\left(\theta_{m}\right), \forall m,
$$

where $\sigma \geq 0$ is a scaling factor. By introducing the notations

$$
\begin{aligned}
& \mathbf{A}=\left[\mathbf{a}\left(\theta_{1}\right), \ldots, \mathbf{a}\left(\theta_{M}\right)\right] \in \mathbb{C}^{N \times M}, \\
& \mathbf{A}_{1}=\left[\mathbf{a}_{1}\left(\theta_{1}\right), \ldots, \mathbf{a}_{1}\left(\theta_{M}\right)\right] \in \mathbb{C}^{N_{R} \times M}, \\
& \mathbf{A}_{2}=\left[\mathbf{a}_{2}\left(\theta_{1}\right), \ldots, \mathbf{a}_{2}\left(\theta_{M}\right)\right] \in \mathbb{C}^{N_{C} \times M},
\end{aligned}
$$

(16) is equivalent to

$$
\operatorname{diag}\left(\mathbf{A}_{2}^{H} \sum_{i=1}^{K} \mathbf{W}_{i} \mathbf{A}_{2}\right)=\sigma \operatorname{diag}\left(\mathbf{A}_{1}^{H} \mathbf{R}_{1} \mathbf{A}_{1}\right),
$$

and the downlink beamforming optimization can thus be formulated as

$$
\begin{array}{ll}
\min _{\sigma, \mathbf{W}_{i}}\left\|\operatorname{diag}\left(\mathbf{A}_{2}^{H} \sum_{i=1}^{K} \mathbf{W}_{i} \mathbf{A}_{2}-\sigma \mathbf{A}_{1}^{H} \mathbf{R}_{1} \mathbf{A}_{1}\right)\right\|^{2} \\
\text { s.t. } & \beta_{i} \geq \Gamma_{i}, \forall i \\
& P_{1} \leq P_{C} \\
& \sigma \geq 0 \\
& \mathbf{W}_{i} \succeq 0, \mathbf{W}_{i}=\mathbf{W}_{i}^{H} \\
& \operatorname{rank}\left(\mathbf{W}_{i}\right)=1, \forall i
\end{array}
$$

where $\Gamma_{i}$ is the SINR threshold of the $i$-th user, $P_{1}$ and $\beta_{i}$ are defined by (2) and (3) while $P_{C}$ is the power budget for downlink communication. It is clear that (19) is non-convex. Nonetheless, a suboptimal solution can be obtained by the classic SDR technique. By omitting the rank-1 constraints, (19) becomes a standard SDP, which can be efficiently solved. For non-rank-1 solutions, an approximated solution is obtained by standard rank-1 approximation techniques, such as eigenvalue decomposition or Gaussian randomization [17].

The proposed zero-forcing optimization can be summarized by the following Algorithm 1 .

Algorithm 1 Zero-forcing Beamforming for Separated Deployment

Input: $\mathbf{H}, \boldsymbol{\Gamma}, P_{0}$, beampattern requirement;

Output: $\mathbf{W}_{i}, \forall i$;

1. Solve (12) or (13) to obtain the radar covariance matrix $\mathbf{R}_{1} \in \mathbb{C}^{N_{R} \times N_{R}}$;

2. Substitute $\mathbf{R}_{1}$ into (19), solve the SDP problem by omitting the rank-1 constraints;

3. Obtain the approximated solution by eigenvalue decomposition or Gaussian randomization.

\section{Beamforming for Shared Deployment}

Although the separated deployment allows flexibility in the design of the radar signal, the full cancellation of the interference inflicted upon the downlink users imposes extra constraints in the radar beampattern design problems, which may result in poor performance. One may also trade-off the radar interference received by users by changing the strict zero-forcing equality constraints to inequalities. However, there are still extra constraints in such problems.

By using the shared deployment, the radar's targets of interest can be viewed as virtual downlink users located in a LoS channel. The beamforming design thus becomes a power sharing problem between the virtual users in the LoS channel and the real users in the fading channel. The difference is that we meet the requirements of the former by using a specific beampattern, and that of the latter by enforcing their SINR constraints. Therefore, the optimization for the shared deployment is to firstly formulate a radar beampattern by solving (9) or (10) with $N_{t}=N$, then substitute the covariance matrix obtained in the communication beamforming problem, which is

$$
\begin{aligned}
\min _{\mathbf{T}_{i}} & \left\|\sum_{i=1}^{K} \mathbf{T}_{i}-\mathbf{R}_{2}\right\|_{F}^{2} \\
\text { s.t. } & \gamma_{i} \geq \Gamma_{i}, \forall i, \\
& \operatorname{diag}\left(\sum_{i=1}^{K} \mathbf{T}_{i}\right)=\frac{P_{0} \mathbf{1}}{N}, \\
& \mathbf{T}_{i} \succeq 0, \mathbf{T}_{i}=\mathbf{T}_{i}^{H}, \operatorname{rank}\left(\mathbf{T}_{i}\right)=1, \forall i,
\end{aligned}
$$

where $\mathbf{R}_{2}$ is obtained by solving (9) or (10), $P_{0}$ and $\gamma_{i}$ are defined as (6) and (7) respectively. Note that the SINR $\gamma_{i}$ 


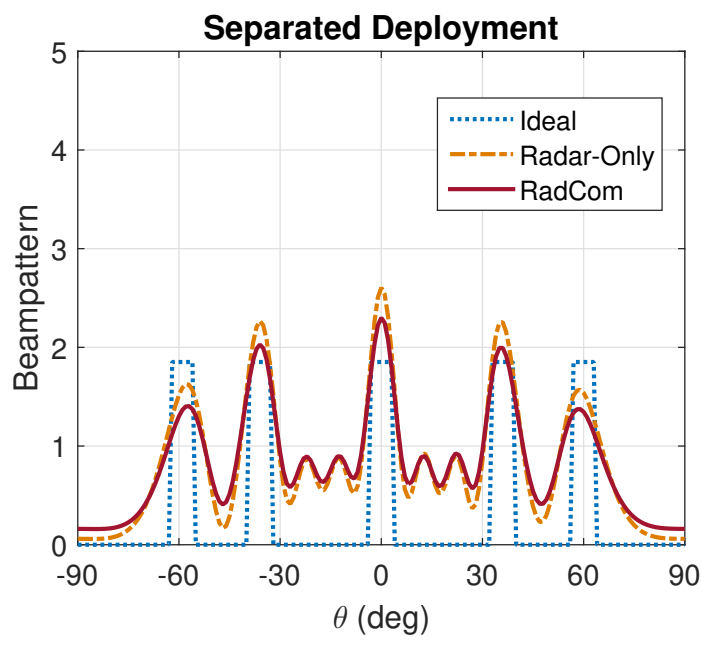

(a)

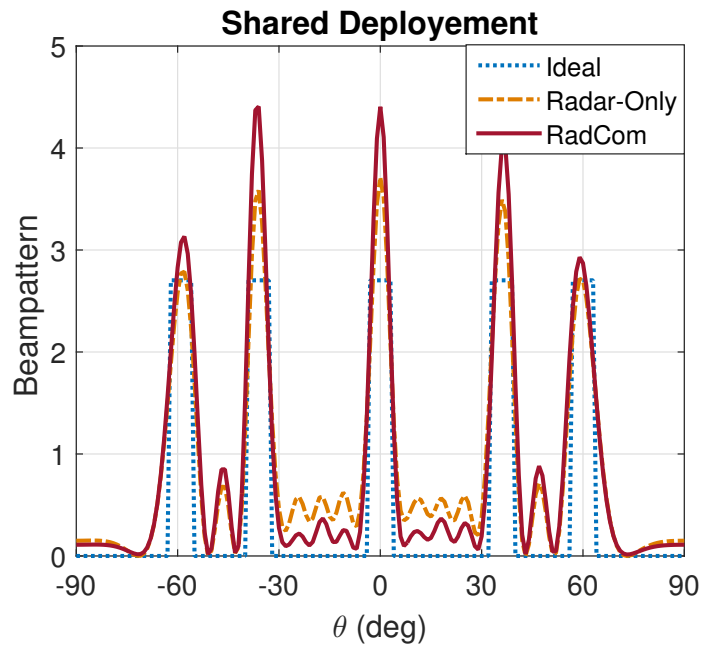

(b)

Fig. 2. Multi-beam beampatterns comparisons for $\Gamma=10 \mathrm{~dB}, K=4$. (a) Separated deployment; (b) Shared deployment.

for the $i$-th user in this case is different from $\beta_{i}$ for the separated deployment above, and that in contrast to (19), all the transmit power is exploited in the above optimization. Here we also employ the equality constraint $(20 \mathrm{c})$ for the power budget, since the radar is often required to transmit at the maximum available power in practice [12]. Similar to (19), the problem (20) can be readily solved using the SDR technique by omitting the rank- 1 constraints. It is worth noting that in both beamforming designs, the achievable data rate is not limited by the radar PRF, and thus results in a higher communication throughput.

\section{Numerical Results}

In this section, numerical results based on Monte Carlo simulations are provided to validate the efficiency of the proposed beamforming approaches. In all the simulations, we set $P_{0}=20 \mathrm{dBm}, N=20, N_{0}=0 \mathrm{dBm}$, and employ a ULA with half-wavelength spacing between adjacent antennas. We also assume that each entry of the channel matrix $\mathbf{H}$ obeys

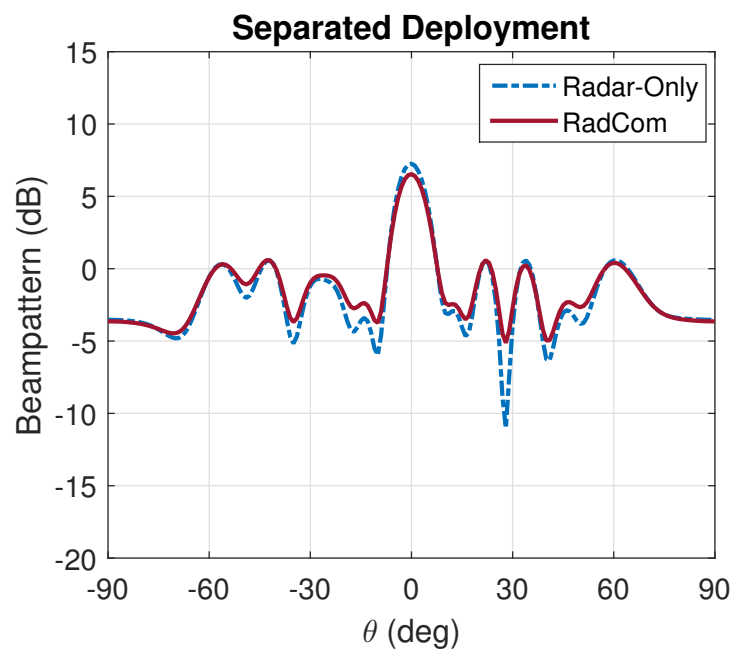

(a)

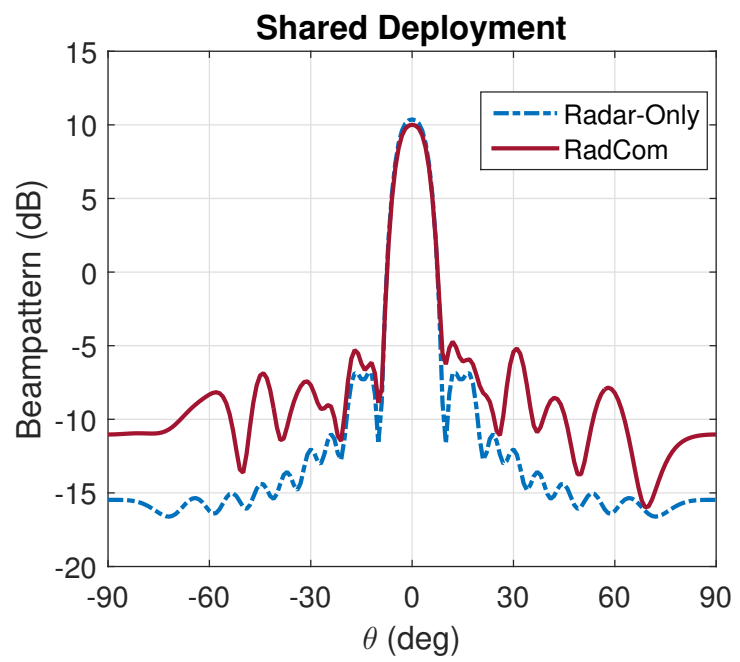

(b)

Fig. 3. $3 \mathrm{~dB}$ beampatterns comparisons for $\Gamma=10 \mathrm{~dB}, K=4$. (a) Separated deployment; (b) Shared deployment.

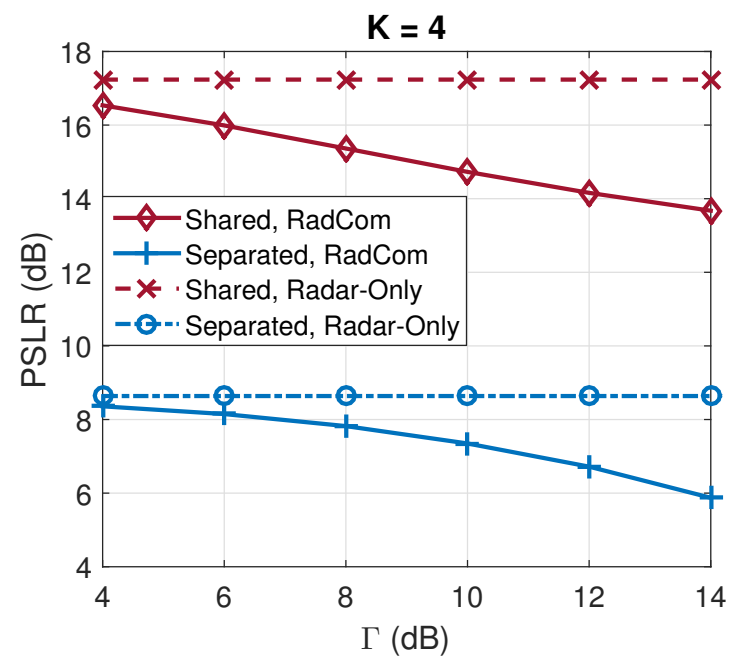

Fig. 4. Trade-off between PSLR and SINR level, $P_{0}=20 \mathrm{dBm}, K=4$. 
the i.i.d. standard complex Gaussian distribution. We solve the beamforming optimizations by the classic SDR technique using the CVX toolbox [18]. From Fig. 2 to Fig. 4, we denote the beampatterns obtained for radar and RadCom joint transmission by 'Radar-Only' and 'RadCom', respectively.

In order to evaluate the performance of the two proposed antenna deployments, we compare the beampatterns obtained by the zero-forcing beamforming and by the shared beamforming. Fig 2 (a) and (b) show the beampatterns with multi-beams, which are originally obtained by solving the problems of (12) and (9) for the radar-only beamforming, and are then formulated by solving (19) and (20) for the two RadCom cases, respectively. The locations of the 5 beams are $\left[-60^{\circ},-36^{\circ}, 0^{\circ}, 36^{\circ}, 60^{\circ}\right]$. The total transmit power, the required SINR for each user and the number of users are set as $\Gamma=10 \mathrm{~dB}, K=4$. For the separated deployment, we set $N_{R}=14, N_{C}=6, P_{R}=P_{C}=P_{0} / 2$. It can be seen that the separated deployment provides a poor beampattern with low peaks at each beam owing to its lower DoF, while the shared deployment achieves a far better one, with even higher peaks than the radar-only beampattern.

In Fig. 3 (a) and (b), we investigate the performance of the two deployments in the case of their $3 \mathrm{~dB}$ beampattern formulations, which are originally obtained by solving problems (13) and (10) for radar only and are then formulated by solving (19) and (20) for the RadCom cases, where the main-beam is centered at $0^{\circ}$ with a $3 \mathrm{~dB}$ width of $10^{\circ}$. All other parameters remain the same as in Fig. 2. Note that the zero-forcing beamforming formulates a RadCom beampattern with a peak-sidelobe-ratio (PSLR) of $7 \mathrm{~dB}$, while the shared beamforming achieves a PSLR of $15 \mathrm{~dB}$.

We finally show in Fig. 4 the trade-off between PSLR and SINR for the resultant $3 \mathrm{~dB}$ beampattern designs of the two deployments, where other parameters are the same as in Fig. 2 and Fig. 3. Once again, we see that for a fixed SINR level, the shared deployment outperforms the separated case leading to a substantial 8dB gain in PSLR.

\section{CONCLUSION}

A novel framework is proposed for the transmit beamforming of the joint RadCom system, where the beamforming schemes are designed to formulate an appropriate radar beampattern, while guaranteeing the SINR and power budget of the communication applications. We have considered both the separated radar and communications antennas deployment and its shared counterpart. Our numerical results show that the shared deployment achieves far better performance than the separated deployment in terms of the trade-off between the quality of the beampattern and the downlink SINR.

\section{ACKNOWLEDGMENT}

This work was supported in part by the Engineering and Physical Sciences Research Council under Project EP/M014150/1, in part by the China Scholarship Council, and in part by the National Natural Science Foundation of China under Project No. 61771047.

\section{REFERENCES}

[1] DARPA. (2016) Shared spectrum access for radar and communications (SSPARC). [Online]. Available: http://www.darpa.mil/program/sharedspectrum-access-for-radar-and-communications

[2] NSF. (2016) Spectrum efficiency, energy efficiency, and security (SpecEES): Enabling spectrum for all. [Online]. Available: https://www.nsf.gov/pubs/2016/nsf16616/nsf16616.htm

[3] R. Saruthirathanaworakun, J. M. Peha, and L. M. Correia, "Opportunistic sharing between rotating radar and cellular," IEEE Journal on Selected Areas in Communications, vol. 30, no. 10, pp. 1900-1910, November 2012.

[4] A. Khawar, A. Abdelhadi, and C. Clancy, "Target detection performance of spectrum sharing MIMO radars," IEEE Sensors Journal, vol. 15, no. 9, pp. 4928-4940, Sept 2015.

[5] B. Li, A. P. Petropulu, and W. Trappe, "Optimum co-design for spectrum sharing between matrix completion based MIMO radars and a MIMO communication system," IEEE Transactions on Signal Processing, vol. 64, no. 17, pp. 4562-4575, Sept 2016.

[6] B. Li and A. Petropulu, "Joint transmit designs for co-existence of MIMO wireless communications and sparse sensing radars in clutter," IEEE Transactions on Aerospace and Electronic Systems, vol. PP, no. 99 pp. 1-1, 2017.

[7] F. Liu, C. Masouros, A. Li, and T. Ratnarajah, "Robust MIMO beamforming for cellular and radar coexistence," IEEE Wireless Communications Letters, vol. 6, no. 3, pp. 374-377, June 2017.

[8] F. Liu, C. Masouros, A. Li, T. Ratnarajah, and J. Zhou, "Interference exploitation for radar and cellular coexistence: The power-efficient approach," arXiv preprint arXiv:1704.08920, 2017.

[9] A. Hassanien, M. G. Amin, Y. D. Zhang, and F. Ahmad, "Dual-function radar-communications: Information embedding using sidelobe control and waveform diversity," IEEE Transactions on Signal Processing, vol. 64, no. 8, pp. 2168-2181, April 2016.

[10] E. BouDaher, A. Hassanien, E. Aboutanios, and M. G. Amin, "Towards a dual-function MIMO radar-communication system," in 2016 IEEE Radar Conference (RadarConf), May 2016, pp. 1-6.

[11] P. M. McCormick, S. D. Blunt, and J. G. Metcalf, "Simultaneous radar and communications emissions from a common aperture, Part I: Theory," in 2017 IEEE Radar Conference (RadarConf), May 2017, pp. 16851690.

[12] P. Stoica, J. Li, and Y. Xie, "On probing signal design for MIMO radar," IEEE Transactions on Signal Processing, vol. 55, no. 8, pp. 4151-4161, Aug 2007.

[13] J. Li and P. Stoica, "MIMO radar with colocated antennas," IEEE Signal Processing Magazine, vol. 24, no. 5, pp. 106-114, Sept 2007.

[14] D. R. Fuhrmann and G. S. Antonio, "Transmit beamforming for MIMO radar systems using signal cross-correlation," IEEE Transactions on Aerospace and Electronic Systems, vol. 44, no. 1, pp. 171-186, January 2008.

[15] S. Sodagari, A. Khawar, T. C. Clancy, and R. McGwier, "A projection based approach for radar and telecommunication systems coexistence," in 2012 IEEE Global Communications Conference (GLOBECOM), Dec 2012, pp. 5010-5014.

[16] J. A. Mahal, A. Khawar, A. Abdelhadi, and T. C. Clancy, "Spectral coexistence of MIMO radar and MIMO cellular system," IEEE Transactions on Aerospace and Electronic Systems, vol. 53, no. 2, pp. 655-668, April 2017.

[17] Z. Q. Luo, W. K. Ma, A. M. C. So, Y. Ye, and S. Zhang, "Semidefinite relaxation of quadratic optimization problems," IEEE Signal Processing Magazine, vol. 27, no. 3, pp. 20-34, May 2010.

[18] M. Grant, S. Boyd, and Y. Ye, "CVX: Matlab software for disciplined convex programming," 2008. 